\title{
Primera identificación en Ecuador de Comamonas kerstersii como agente infeccioso
}

\section{First identification in Ecuador of Comamonas kerstersii as an infectious agent}

\author{
Galo Farfán-Cano ${ }^{1,2,3}$, Henry Parra-Vera ${ }^{3,4}$, Ana Ávila-Choez ${ }^{1,3,5}$, Glen Silva-Rojas ${ }^{6,}$ y Stanley Farfán-Cano ${ }^{1,2}$
}

\author{
'Sociedad de Infectología del Guayas. \\ Universidad Rey Juan Carlos - España. \\ ${ }^{3}$ Hospital General del Norte de Guayaquil Los Ceibos. \\ ${ }^{4}$ Centro de Investigación Microbiológica (CIM), Guayaquil-Ecuador. \\ ${ }^{5}$ Pontificia Universidad Católica Argentina. \\ שUniversidad Católica Santiago de Guayaquil, Guayaquil-Ecuador.
}

Sin financiamiento

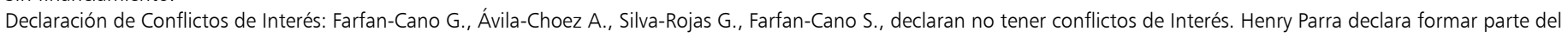
CIM, como investigador principal del mismo.

Recibido: 23 de julio de 2019 / Aceptado: 28 de marzo de 2020

\section{Resumen}

Comunicamos la primera identificación en Ecuador del microorganismo Comamonas kerstersii, cepa aislada a partir de una muestra de líquido de la cavidad abdominal e identificada mediante la técnica de espectrometría de masas MALDITOF. Fue obtenida durante el acto quirúrgico de un paciente con el diagnóstico de una peritonitis aguda, secundaria a una apendicitis perforada. Este microorganismo es considerado un patógeno poco común. Pese a que el género al que pertenece tiene una amplia distribución geográfica, es considerado un agente oportunista.

Palabras clave: Infecciones intraabdominales; apendicitis; Comamonas; espectrometría de masa por láser de matriz asistida de ionización desorción; microbiología.

\section{Introducción}

$\mathrm{E}$ 1 género Comamonas pertenece a la familia Comamonadaceae. Corresponden a $\beta$ proteobacterias en el grupo de homología de ARNr de Pseudomonas III. Son bacilos gramnegativos, aerobios, móviles, pigmentados en rosa, oxidasa positivos, que crecen bien en medios bacteriológicos de rutina. Las especies más frecuentemente identificadas son Comamonas terrigena, C. testosteroni, C. denitrificans y C. nitrativorans. Usualmente este género tiene una baja virulencia, y ocasionalmente originan

\begin{abstract}
We report the first identification in Ecuador of the microorganism Comamonas kerstersii, a strain isolates from a sample of fluid from the abdominal cavity and identified by the technique of mass spectrometry MALDITOF. It was obtained during the surgical act of a patient with the diagnosis of acute peritonitis, secondary to a perforated appendicitis. This microorganism is considered a rare pathogen. Even though the genus to which it belongs has a wide geographical distribution, this pathogen is considered an opportunistic agent.

Keywords: Intraabdominal infections; appendicitis; Comamonas; spectrometry mass, matrix-assisted laser desorption-ionization; microbiology.
\end{abstract}

infecciones en humanos ${ }^{1,2}$

La especie Comamonas testosteroni (anteriormente Pseudomonas testosteroni) es el patógeno del género que más comúnmente se aisla en la mayoría de los casos, debido a la limitada capacidad de los laboratorios clínicos para la correcta identificación de las especies de Comamonas. En algunos casos pueden ser reportados como bacilos gramnegativos, no fermentadores ${ }^{2}$. En un estudio de biodiversidad en los bosques nativos Llucud y Palictahua de la provincia de Chimborazo en 2018, se identificaron especies de Comamonas humi y C. badia en el medio ambiente ${ }^{3}$. 


\section{Caso clínico}

Paciente masculino de 14 años, que ingresó por un cuadro de dolor abdominal de tres días de evolución. Posterior a la evaluación por el cirujano pediátrico se diagnosticó un abdomen agudo, por lo que fue sometido a una intervención quirúrgica por una peritonitis aguda secundaria a una apendicitis. Se realizó una apendicectomía convencional por vía laparoscópica, con hallazgos quirúrgicos de un apéndice cecal gangrenado de localización pélvica, perforado en la base apendicular, ciego e íleon terminal con áreas de fibrina. Se encontraron aproximadamente $200 \mathrm{ml}$ de líquido purulento libre en la cavidad, del cual se tomaron muestras para estudio microbiológico en el laboratorio de la unidad asistencial.

El procesamiento de la muestra se efectuó mediante la siembra en diferentes medios de cultivos (agar sangre, agar MacConkey y agar chocolate). Se incubaron a $35^{\circ}$ $\pm 2^{\circ} \mathrm{C}$ por $24 \mathrm{~h}$ en atmósfera aerobia. A las 24 h se observó el desarrollo de colonias en toda la placa. Se procedió a realizar la identificación y prueba de sensibilidad mediante un método automatizado con el equipo Phoenix 50, estableciéndose la identificación preliminar de Comamonas testosteroni. Posteriormente se envió la cepa al Centro de Investigación Microbiológica para la confirmación de género y especie.

Para la identificación de la especie bacteriana, se recibió en el laboratorio de referencia una cepa microbiana de 8 a 10 h de desarrollo en agar sangre, con una identificación presunta de Comamonas testosteroni. La misma cepa fue procesada para su análisis por técnica de espectrometría de masas (MS) matrix-assisted laser desorption ionization time-of-flight (MALDITOF), en el equipo MALDI Biotyper de Bruker (versión 3.4), que finalmente identificó la cepa como Comamonas kerstersii con un spectral score de 2,83 .

El paciente recibió inicialmente un esquema empírico con ceftriaxona, metronidazol y amikacina, durante los cinco días siguientes de la cirugía. Ante una evolución estacionaria, con la herida con signos de infección, se cambió a piperacilina/tazobactam en asociación con amikacina. Al séptimo día del nuevo esquema, y con el resultado preliminar del género Comamonas, se procedió a mantener el esquema con piperacilina/tazobactam hasta completar 14 días de tratamiento. Con el resultado definitivo de Comamonas kerstersii, y una evolución lenta de la herida, fue finalmente dado de alta a los 29 días del acto operatorio.

\section{Discusión}

El género Comamonas pertenece al orden Burkholderiales, y son microorganismos quimioheterótrofos aeróbicos, anaeróbicos facultativos. Tienen una amplia distribución geográfica y se encuentran comúnmente en el suelo, las plantas, los animales, los saprófitos acuáticos y el agua; rara vez se asocian a infecciones en humanos, aunque han sido asociados a infecciones intraabdominales complicadas con perforación apendicular. Algunas especies (en particular C. testosteroni, C. kerstersii y $C$. aquatica) han sido relacionadas con infecciones invasoras

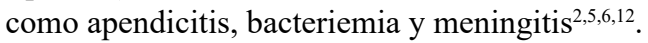

Comamonas kerstersii fue descrita como uno de tres grupos genotípicamente separados de $C$. terrígena en 2003. Los reportes de Comamonas relacionados con infecciones en humanos han aumentado desde 2013, siendo principalmente asociadas con perforación apendicular ${ }^{6}$. Particularmente, se ha referido la asociación de la especie C. testosteroni con apendicitis perforada ${ }^{4,5}$.

Cabe resaltar que, en la mayoría de los reportes presentados en la literatura científica, la identificación del microorganismo $C$. testosteroni se efectuó mediante métodos fenotípicos en cuyas bases de datos no se encuentra el registro de $C$. kerstersii. Este es el caso de las bases de datos de los sistemas VITEK, ATB, API, Siemens y BD, que solo cuentan con dos tipos de registro de Comamonadaceae (C. acidovorans y C. testosteroni), lo que puede subestimar el diagnóstico de C. kerstersii ${ }^{4-6}$.

La desorción/ionización mediante láser asistida por matriz (MALDI por sus siglas en inglés), acoplada a un analizador de tiempo de vuelo (TOF por sus siglas en inglés), es una técnica de ionización suave, aplicada en espectrometría de masas (MS por sus siglas en inglés) para el análisis de biomoléculas y moléculas orgánicas grandes, que tienden a hacerse frágiles y fragmentarse cuando son ionizadas por métodos más convencionales ${ }^{7}$.

Algunas nuevas técnicas, como MALDI-TOF-MS y la secuenciación de genes mediante la reacción de la polimerasa en cadena (RPC) de la $16 \mathrm{~S} \mathrm{ARNr}$, pueden confirmar las especies específicas de Comamonas.

En la actualidad, la mayoría de los casos de aislamientos de $C$. kerstersii comunicados alrededor del mundo, han sido identificados por MALDI-TOF-MS. Es un método rápido y preciso para diferenciar entre las especies de $\mathrm{Co}$ mamonas, por lo cual es el método que debería utilizarse para identificar la especie ${ }^{8}$.

La identificación bacteriana directamente de colonias y muestras mediante MALDI-TOF, se ha descrito como una herramienta revolucionaria perfectamente adaptada al laboratorio de microbiología clínica, utilizada para identificar especies y subespecies bacterianas, y en caso de brotes, esta técnica es capaz de permitir la identificación de los linajes de las cepas ${ }^{5-11}$.

$\mathrm{Wu}$ y cols. ${ }^{12}$ en un estudio del género Comamonas, proporcionaron información genómica acerca de los posibles roles de virulencia mediante el análisis del conjunto completo de genes y el genoma central, y encontraron que 
los factores de virulencia en Comamonas eran altamente específicos de cada especie.

Comamonas kerstersii tiende a ser susceptible a antibacterianos $\beta$-lactámicos combinados con inhibidores de betalactamasas, quinolonas, cefalosporinas de cuarta generación, aminoglucósidos, carbapenémicos y polimixinas ${ }^{9}$; también se ha mencionado una aparente sensibilidad a cefalosporinas de primera, segunda y tercera generación ${ }^{1,3-6,8}$.

En los reportes en la literatura especializada, se ha comunicado una respuesta clínica favorable a $\beta$-lactámicos con inhibidores de betalactamasas (ampicilina/sulbactam o amoxicilina/ácido clavulánico en casos sin complicaciones, y piperacilina/tazobactam en casos complicados) por lo cual se sugiere el uso de estos agentes como alternativas terapéuticas de primera línea.

En casos de alergia reportada a $\beta$-lactámicos, las quinolonas son una alternativa, ya que tienen efecto contra otras bacterias anaerobias. En casos complicados y pacientes inmunocomprometidos, pudiera considerase el uso de cefalosporinas de tercera o cuarta generación o carbapenémicos, especialmente en casos de coinfección por otros microorganismos que pudieran presentar resistencia a antibacterianos.

\section{Conclusión}

El género Comamonas tiene una amplia distribución global en varios nichos ecológicos. Existen reportes esporádicos de especies de este género relacionados a infecciones en humanos. Sin embargo, es de notar que las técnicas microbiológicas convencionales y algunos sistemas automatizados fenotípicos pudieran ocasionar un sesgo en la identificación correcta de la especie como agente etiológico de ciertas infecciones. Cabe resaltar que la técnica MALDI-TOF, pese a no tener registros hasta el momento de cepas de $C$. kerstersii con resistencia a antimicrobianos, es una de las opciones que permiten establecer el diagnóstico definitivo por este microorganismo.

\section{Referencias bibliográficas}

1.- Farooq S, Farooq R, Nahvi N. Comamonas testosteroni: Is it still a rare human pathogen? Case Rep Gastroenterol 2017; 11: 42-7. doi: 10.1159/000452197.

2.- Steinberg J, Burd E. Otros bacilos gramnegativos y gramvariables. Comamonas y especies de Delftia. In: Bennett J, Dolin R, Blaser M, ed. by. Mandell, Douglas, y Bennett, Principios y Práctica de Enfermedades Infecciosas de Bennett. 8th ed. Barcelona: Elsevier; 2015; p. 2667-83.

3.- Patiño-Castillo B. Determinación de la biodiversidad microbiana de los bosques nativos Llucud y Palictahua de la provincia de Chimborazo 2018 Bachelor's thesis, Escuela Superior Politécnica de Chimborazo. Fecha de acceso: 22 de junio de 2019. Disponible en: http://dspace.espoch.edu.ec/bitst ream/123456789/8766/1/33T0188.pdf.

4.- Reddy A K, Murthy S I, Jalali S, Gopinathan U. Post-operative endophthalmitis due to an unusual pathogen, Comamonas testosteroni.
J Med Microbiol 2009; 58: 374-5. doi: 10.1099/jmm.0.006072-0.

5.- Almuzara M N, Cittadini R, Vera Ocampo C, Bakai R, Traglia G, Ramirez MS, et al. Intra-abdominal infections due to Comamonas kerstersii. J Clin Microbiol 2013; 51: 19982000. doi: 10.1128/JCM.00659-13.

6.- Zhou Y, Ma H, Dong Z, Shen M. Comamonas kerstersii bacteremia in a patient with acute perforated appendicitis. Medicine (Baltimore) 2018; 97: e9296 doi: 10.1097/ MD.0000000000009296.

7.- Plascencia-Villa G. Espectometría de masas. Maestría en Ciencias Bioquímicas, Instituto de Biotecnología, Universidad Nacional Autónoma de México, junio de 2003. Fecha de acceso: 22 de junio de 2019. Disponible en: http://www.ibt. unam.mx/computo/pdfs/met/Spec_Masas.pdf

8.- Biswas J, Fitchett J, O'Hara G. Comamonas kerstersii and the perforated appendix. J Clin Microbiol 2014; 52: 3134. doi: 10.1128/ JCM.00909-14.

9.- Seng P, Abat C, Rolain J M, Colson P, Lagier $\mathrm{J} \mathrm{C}$, Gouriet F, et al. Identification of rare pathogenic bacteria in a clinical microbiology laboratory: impact of matrix-assisted laser desorption ionization-time of flight mass spectrometry. J Clin Microbiol 2013; 51: 2182 94. doi: 10.1128/JCM.00492-13.

10.- Bittar F, Cassagne C, Bosdure E, Stremler N, Dubus J, Sarles J, et al. Outbreak of Corynebacterium pseudodiphtheriticum infection in cystic fibrosis patients, France. Emerg Infect Dis 2010; 16: 1231-6. doi: 10.3201/eid1608.100193.

11.- Griffin P M, Price G R, Schooneveldt J M, Schlebusch S, Tilse M, Urbanski T, et al. Use of matrix-assisted laser desorption ionization-time of flight mass spectrometry to identify vancomycin-resistant Enterococci and investigate the epidemiology of an outbreak. J Clin Microbiol 2012; 50: 2918-31. doi: 10.1128/JCM.01000-12.

12.- Wu Y, Zaiden N, Cao B. The core- and pangenomic analyses of the genus Comamonas: from environmental adaptation to potential virulence. Front Microbiol 2018; 9: 3096. doi: 10.3389/fmicb.2018.03096. 